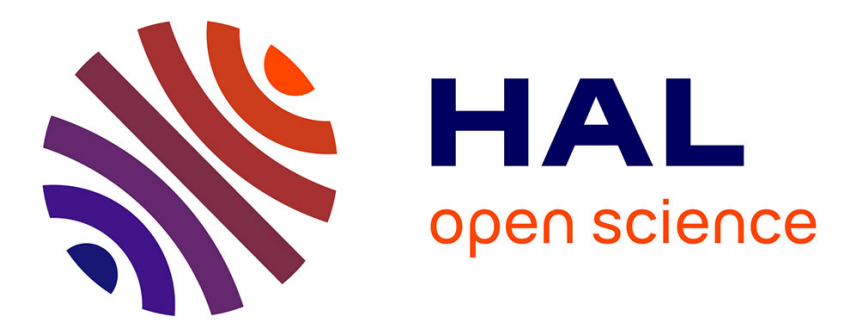

\title{
Défendre les nationalistes algériens en lutte pour l'indépendance. La " défense de rupture " en question Sylvie Thénault
}

\section{To cite this version:}

Sylvie Thénault. Défendre les nationalistes algériens en lutte pour l'indépendance. La " défense de rupture " en question. Le Mouvement social, 2012, 240 (3), pp.121. 10.3917/lms.240.0121 . hal02355609

\author{
HAL Id: hal-02355609 \\ https://hal.science/hal-02355609
}

Submitted on 12 Nov 2019

HAL is a multi-disciplinary open access archive for the deposit and dissemination of scientific research documents, whether they are published or not. The documents may come from teaching and research institutions in France or abroad, or from public or private research centers.
L'archive ouverte pluridisciplinaire HAL, est destinée au dépôt et à la diffusion de documents scientifiques de niveau recherche, publiés ou non, émanant des établissements d'enseignement et de recherche français ou étrangers, des laboratoires publics ou privés. 


\title{
DÉFENDRE LES NATIONALISTES ALGÉRIENS EN LUTTE POUR L'INDÉPENDANCE. LA « DÉFENSE DE RUPTURE » EN QUESTION
}

\author{
Sylvie Thénault
}

La Découverte | « Le Mouvement Social »

2012/3 n² 240 | pages 121 à 135

ISSN 0027-2671

ISBN 9782707174499

Article disponible en ligne à l'adresse :

https://www.cairn.info/revue-le-mouvement-social-2012-3-page-121.htm

Distribution électronique Cairn.info pour La Découverte.

(C) La Découverte. Tous droits réservés pour tous pays.

La reproduction ou représentation de cet article, notamment par photocopie, n'est autorisée que dans les limites des conditions générales d'utilisation du site ou, le cas échéant, des conditions générales de la licence souscrite par votre établissement. Toute autre reproduction ou représentation, en tout ou partie, sous quelque forme et de quelque manière que ce soit, est interdite sauf accord préalable et écrit de l'éditeur, en dehors des cas prévus par la législation en vigueur en France. Il est précisé que son stockage dans une base de données est également interdit. 


\title{
Défendre les nationalistes algériens en lutte pour l'indépendance. La " défense de rupture " en question
}

\author{
par Sylvie ThénAult*
}

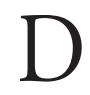

ans l'histoire de l'engagement des avocats, la " défense de rupture ", incarnée par Jacques Vergès, est considérée comme l'héritage principal de la guerre d'indépendance algérienne ${ }^{1}$. La " maturation " de la notion de "défense de rupture ", précise son sulfureux représentant, se fit "progressivement, au fur et à mesure du développement ${ }^{2}$ de la guerre. L'expression, du reste, n'est pas repérable pendant les années du conflit elles-mêmes. En 1961, les avocats du collectif du Front de libération nationale (FLN), dont Jacques Vergès faisait partie, parlaient de " défense politique " ${ }^{3}$. Ils la présentaient comme la réponse logique aux procès " politiques ", dont l'enjeu était " la condamnation d'un ordre politique au nom d'un autre " ${ }^{4}$; leur enjeu dépassait donc les circonstances et la personnalité des accusés. Dans ce contexte, les juges n'auraient eu que faire de la vérité des faits ni des hommes conduits dans le prétoire. Ces derniers, de leur côté, n’auraient rien eu à perdre en se comportant comme des "soldats" au service d'une cause, comme des "militants " revendiquant leurs actes. Cette " défense politique " se voulait " originale ", " offensive ", " anticolonialiste ", disaient ces avocats. La rupture avec les normes et les règles du procès était de l'ordre du possible, mais elle n'était pas préconisée ni nommée comme telle.

La stratégie de rupture ne fut couchée sur le papier par Jacques Vergès qu'en 1968, dans De la stratégie judiciaire. Il y distinguait la " défense de connivence » et la "défense de rupture ». La " connivence » se manifeste par le respect de l'institution judiciaire, le traitement sérieux des questions de procédure, la volonté de dialoguer avec le tribunal. La " rupture " suppose tout le contraire : à elle, l'irrévérence, les incidents, le scandale. La différence, néanmoins, n’a rien d'absolu. Les attitudes du défenseur, expliquait Jacques Vergès, se situent sur un continuum riche de toutes les possibilités, de la connivence totale à la rupture radicale, en passant par toutes les combinaisons imaginables. Il déconnectait par ailleurs chaque type de défense de la nature du procès : la " connivence " et la " rupture " peuvent exister toutes deux en droit commun comme en matière politique. Par la suite, il cita Jacques Mesrine, liant ses méfaits à la contestation de l'ordre politique et social, en exemple d'une « défense de rupture " pratiquée dans des affaires purement criminelles ${ }^{5}$. Quoi qu'il en soit,

* Directeur de recherche au CNRS, Centre d'histoire sociale du XX'e siècle (UMR 8158).

1. L. IsraËL, L'arme du droit, Paris, Presses de Sciences Po, 2009.

2. J. Vergès, De la stratégie judiciaire, Paris, Éditions de Minuit, 1968, p. 188.

3. A. Benabdallah, M. Courrégé, M. Oussedik, J. Vergès, M. Zavrian, Défense politique, Paris, François Maspero, Cahiers libres, nº 15, 1961.

4. Ibid., p. 9 .

5. J. Vergès, Le salaud lumineux : conversations avec Jean-Louis Remilleux, Paris, Michel Laffont, 1990 , p. 155-156. 
il fallut quelques années après l'indépendance de l'Algérie pour que la « défense de rupture " soit explicitée sous une forme aboutie, appelée à faire école.

Jacques Vergès souligne également que, s'il a " conceptualisé cette notion ", il ne l'a pas "inventée " ${ }^{6}$. Dans De la stratégie judiciaire, il remonte jusqu'aux procès de Socrate et de Louis XVI mais retient surtout celui de Dimitrov, accusé de l'incendie du Reichstag en 1933. L'avocat communiste Marcel Willard, s'inspirant de Lénine, avait alors défini la défense à mettre en ouvre en des termes que Jacques Vergès faisait siens et que les avocats du collectif FLN avaient déjà cités en 1961 : «D’abord, prendre et garder l'initiative ; à la faveur de cette initiative, concevoir et exécuter un plan stratégique ; démolir politiquement, non pas seulement l'accusation, mais l'ennemi lui-même. Le rendre ridicule $"^{7}$. En tant qu'ancien militant du PCF, Jacques Vergès puisait évidemment dans sa propre formation politique. À travers Marcel Willard et en citant aussi Lénine, il inscrivait la "défense de rupture " dans une généalogie communiste. Le lien tissé entre la genèse de cette défense et le moment de la guerre d'indépendance algérienne s'en trouve distendu.

Il reste indéniable que cette guerre constitua un moment majeur dans la formalisation de la "défense de rupture ", même si ce type de défense résulte d'une évolution entamée en amont et terminée en aval. Cette réinscription de l'élaboration de la "défense de rupture " dans une dynamique historique ouvre cependant deux questions. Elle pose d'abord celle des précédents à la période de la guerre d'indépendance : de quelles expériences les avocats engagés aux côtés des nationalistes algériens bénéficiaient-ils au moment du déclenchement de cette guerre ? Selon Sharon Elbaz, la défense des nationalistes du Rassemblement démocratique africain (RDA), entre 1949 et 1952, a constitué un "banc d'essai pour les collectifs d'avocats en guerre d'Algérie " ${ }^{8}$; il rappelle également qu'en Algérie même, avant 1954, les procès consécutifs au démantèlement de l'Organisation spéciale (OS), branche armée du mouvement nationaliste, avaient mobilisé des avocats engagés, inscrits aux barreaux locaux ou venus de métropole?. À la généalogie communiste s’ajoute ainsi une généalogie coloniale.

Par ailleurs, si la "défense de rupture " n'était qu'en cours de " maturation " entre 1954 et 1962, la question de savoir comment les nationalistes algériens furent défendus mérite d'être posée. Il faut, pour la résoudre concrètement, tenter de pénétrer dans les salles d'audience et faire revivre les débats des procès - une gageure ? La tentative, en tout cas, conduit à distinguer les conditions d'exercice de la défense en France métropolitaine et en Algérie. Il importe de préciser d'emblée les limites de l'action du collectif dont Jacques Vergès est resté le symbole. Il s'agissait en réalité du collectif de la Fédération de France du FLN, qui n’avait pas pour vocation prioritaire d'intervenir en Algérie. En outre, il ne fut formé qu’en 1958, plus de trois ans après le déclenchement de l'insurrection et le déploiement d'une répression judiciaire d'envergure.

6. Ibid., p. 157.

7. ID., De la stratégie judiciaire, op. cit., p. 107.

8. S. ElbAZ, «Les avocats métropolitains dans les procès du Rassemblement démocratique africain (1949-1952) : un banc d'essai pour les collectifs d'avocats en guerre d'Algérie ? ", Bulletin de l'IHTP, $n^{\circ} 80,2002$, en ligne, URL : http://www.ihtp.cnrs.fr/spip.php\%3Farticle350\&lang=fr.html.

9. S. Elbaz, "L'avocat et sa cause en milieu colonial. La défense politique dans le procès de l'Organisation spéciale (OS) du Mouvement pour le triomphe des libertés démocratiques (MTLD) en Algérie (1950-1952) ", Politix, vol. 16, n62, 2003, p. 65-91. 


\section{Le réinvestissement du " collectif » comme forme d'organisation}

La répression judiciaire frappa d'abord sur le sol algérien. Dès avril 1955, la loi d'état d'urgence remit aux tribunaux militaires le jugement des crimes. Les cours d'assises étaient dessaisies à leur profit. Les tribunaux correctionnels, quant à eux, restaient compétents pour juger les délits. Des milliers de procédures, ouvertes pour " atteinte à la sûreté de l'État ", " association de malfaiteurs ", " coups et blessures ", « incendie volontaire ", " assassinat "... s'accumulèrent dans les cabinets d'instruction ; plus de quatre mille étaient en cours en décembre 1955. Les tribunaux correctionnels jugeaient déjà des centaines d'accusés par mois - 616 en décembre 1955 - et les tribunaux militaires plusieurs dizaines, voire plus de cent. Puis la répression s'amplifia. Le nombre d'accusés dépassa le millier au début de l'année 1957 : entre janvier 1957 et mai 1958, les tribunaux correctionnels jugeaient huit cents à mille personnes par mois, tandis que cinq cents personnes en moyenne étaient renvoyées, chaque mois également, devant les tribunaux militaires ${ }^{10}$. Ces hommes et ces femmes provenaient d'organisations diverses. Il s'agissait de membres du FLN, mais aussi de son concurrent le Mouvement national algérien (MNA) ou encore du Parti communiste algérien (PCA).

Les premiers avocats métropolitains intervenant dans ce contexte furent les avocats dits " historiques", en raison de l'ancienneté de leur engagement contre la répression judiciaire coloniale ${ }^{11}$. Leur engagement remontait en effet aux lendemains de la Seconde Guerre mondiale, lorsque trois grandes vagues de répression les avaient mobilisés. Avant celles étudiées par Sharon Elbaz, visant les militants du RDA en Afrique et les membres de l'OS en Algérie, celle des insurgés de Madagascar en 1947 avait ouvert cette séquence anti-coloniale. Ces trois vagues répressives avaient conduit des avocats parisiens de diverses obédiences à s'organiser collectivement pour assurer la défense des accusés devant les tribunaux mais aussi pour médiatiser leur cause. Les avocats Pierre et Renée Stibbe, très engagés dans la défense des Malgaches, avaient impulsé en métropole la formation d'un Comité pour l'amnistie des condamnés politiques d'Outre-mer, soutenu par Claude Bourdet et s'inscrivant dans la mouvance intellectuelle de France Observateur. Progressivement, ce Comité glissa de la cause malgache à la cause algérienne. En avril 1955, il publiait son premier communiqué consacré aux condamnés algériens : se déclarant "inquiet de constater qu'un grand nombre de militants politiques algériens sont emprisonnés alors que seule une action politique normale leur est reprochée ". Le Comité demandait leur libération et dénonçait " les mesures d'interdiction de séjour, de censure de la presse et de traduction de civils devant les tribunaux militaires par application de l'état d'urgence ${ }^{12}$. En novembre 1955, ce même Comité organisait une réunion publique pour la grâce des condamnés à mort algériens au cours de laquelle Louis Massignon, Claude Bourdet mais aussi l'avocat Yves Dechézelles prirent la parole ${ }^{13}$.

10. S. ThÉnault, Une drôle de justice, les magistrats dans la guerre d'Algérie, Paris, La Découverte, 2001.

11. L’expression « avocats historiques » serait de Pierre Stibbe. Voir S. ElbaZ, « Les avocats métropolitains... ", art. cité.

12. Communiqué du Comité pour l'amnistie des condamnés politiques d'outre-mer, France Observateur, 28 avril 1955.

13. D'après Le Monde du 9 novembre 1955. 
La répression du RDA, quant à elle, avait suscité la création d'un " collectif ", sous l'égide du communiste Henri Douzon. Ce "mode spécifique d'organisation " était de nature "politico-professionnelle ${ }^{14}$, dans la mesure où il naquit d'une double conjoncture. Au plan professionnel, il fallait prendre en charge la défense des accusés du RDA dans un contexte où les barreaux locaux, en Afrique même, ne pouvaient l'assurer pour diverses raisons : effectifs insuffisants, statuts contraignants limitant la liberté d'expression des défenseurs, étroitesse des milieux juridique et judiciaire en contexte colonial, propice à des relations de connivence entre acteurs de tous bords. L'activité du collectif formé en métropole dépassait cependant les limites de la mission professionnelle des avocats. Ces derniers étaient appelés à s'engager sur le terrain politique. Non seulement les audiences constituaient autant de tribunes au profit de la dénonciation de la colonisation, mais les avocats prirent aussi en charge la médiatisation de la cause du RDA et acceptèrent de mettre leurs facilités de communication avec les détenus au service de l'organisation politique africaine. Le collectif agissait en concertation directe avec le RDA. Leurs relations étaient favorisées par l'adhésion au communisme de part et d'autre - au début du moins. La majorité des avocats du collectif étaient membres du PCF. Marcel Willard lui-même plaida dans le plus important des procès de cette période. En outre, c'était le Secours populaire qui finançait leurs activités. Les élus du RDA au Parlement, quant à eux, furent apparentés au groupe communiste jusqu'en octobre 1950. Mais à cette date, ils prirent leur autonomie. Cette rupture se manifesta par des réticences nouvelles à l'égard des avocats communistes. La direction du RDA appela à les "déconstituer ", c'est-à-dire que leurs clients devaient les remplacer par d'autres avocats ${ }^{15}$. Ce moment de la répression du RDA préfigure largement celui de la guerre d'indépendance algérienne, dans ses diverses problématiques : mise en place d'un réseau d'avocats se relayant dans la prise en charge des affaires, défense politique, engagement des défenseurs au-delà de leur mission professionnelle, liaison éventuellement conflictuelle avec l'organisation visée par la répression.

Simultanément à leurs interventions en Afrique, les membres du collectif formé dans les circonstances de la répression du RDA s'engagèrent en terre algérienne. En mars 1950, le démantèlement de l'OS avait conduit à l'arrestation de 363 nationalistes, dont 195 furent condamnés ${ }^{16}$. Les époux Stibbe, Henri Douzon, Pierre Braun, Paul Vienney traversèrent la Méditerranée dès cette époque. Ils venaient seconder les avocats locaux, dont certains étaient eux-mêmes militants du PPA-MTLD ${ }^{17}$, l'organisation nationaliste alors existante ; l'OS démantelée, dont les membres étaient poursuivis, en était la branche armée. Le trotskiste Yves Dechézelles jouait auprès du PPA-MTLD le même rôle qu'Henri Douzon auprès du RDA. Les deux hommes servaient d' " interface " ${ }^{18}$ entre le collectif de défenseurs et l'organisation politique pour laquelle ils agissaient. Dans le cas du PPA-MTLD, le choix d'un trotskiste marquait une prise de distance avec le PCF, même si l'intervention d'avocats communistes ne posait pas problème. Pierre Braun se souvient de "réunions

14. S. ElbAZ, «Les avocats métropolitains... ", art. cité.

15. Le néologisme "déconstituer " est conçu comme le contraire de " constituer » un avocat (le désigner comme défenseur dans une affaire).

16. Ch.-R. Ageron, Histoire de l'Algérie contemporaine, t. II, Paris, PUF, 1979, p. 590.

17. Parti du peuple algérien - Mouvement pour le triomphe des libertés démocratiques. Le MTLD était la vitrine légale du PPA, interdit, d'où cette appellation.

18. S. Elbaz, "L'avocat et sa cause en milieu colonial... ", art. cité. 
pour organiser la défense au siège parisien du MTLD qui se trouvait dans le quartier Saint-Michel ${ }^{19}$. Comme l'explique lui-même Jacques Vergès, «les nationalistes faisaient appel aux avocats du PC car la politique coloniale de ce parti était différente de celle des autres partis de gauche : c'était le seul parti qui était contre la répression coloniale ${ }^{20}$.

Le collectif créé dans les circonstances de la répression en Afrique forma ainsi un réseau d'avocats anticolonialistes, mobilisables le cas échéant. Identifié à l'époque comme le collectif du Secours populaire, il restait sous la houlette d'Henri Douzon. Le $1^{\text {er }}$ novembre 1954 , le déclenchement de l'insurrection algérienne le mobilisa de nouveau, les militants emprisonnés faisant appel à eux. "Les nationalistes avaient en mémoire le nom de ces avocats ", reconnaît Mourad Oussedik, qui fut la cheville ouvrière de la création du collectif de la Fédération de France du FLN en $1958^{21}$. D’après Pierre Kaldor, ancien responsable du Secours populaire, qui plaida luimême en faveur des nationalistes africains, Henri Douzon «travaillait en liaison très directe et continue avec un secteur de la direction du PC qui tenait très fortement à ce que la défense soit assurée, aussi bien pour les communistes que pour les gens du Front qui sollicitaient notre appui " ${ }^{22}$. Mais cette " petite équipe », pour reprendre l'expression de Pierre Braun, fut vite submergée et de nombreux autres avocats s'y agrégèrent. Pierre Kaldor qui fut sollicité pour la première fois en 1956, alors que " tout le monde était en vacances », témoigne :

"Ils se sont partagés la défense de ceux qui se trouvaient arrêtés et cela, quelle que soit l'opinion des personnes arrêtées. Il y avait des gens du FLN, du PCA, des syndicalistes aussi. Et au fur et à mesure que la répression s'est étendue, ces avocats, qui avaient besoin de penser aussi au fonctionnement de leur cabinet à Paris, ont appelé d'autres avocats, ponctuellement, pour leur donner un coup de main ${ }^{23}$.

Au cours de l'année 1957, Pierre Kaldor prit la suite de son ami Henri Douzon, contraint d'abandonner la coordination du collectif pour des raisons de santé. Son travail consistait à organiser le départ de ses confrères se relayant en Algérie : "Les inculpés avaient désigné des avocats qu'ils connaissaient, untel ou untel, et c'est cet avocat désigné par l'inculpé qui était convoqué par le juge d'instruction. Mais si l'avocat qui avait été désigné ne pouvait pas partir, c'était un autre qui partait avec un bon de substitution du premier ». Pierre Kaldor avait ainsi connaissance d'une dizaine d'avocats sur lesquels il pouvait compter pour des départs réguliers, mais il lui arrivait aussi de faire appel à d'autres confrères en cas de besoin. La liste des avocats qui sont allés plaider en Algérie est donc longue : Henri Douzon, Pierre Braun, Pierre Kaldor lui-même bien sûr, mais aussi Léo Matarasso, Charles Lederman, Joë Nordmann, Jules Borker, Marie-Louise Jacquier-Cachin, Roger Cevaer, Nicole

19. P. Braun, entretien avec l'auteur. Comme tous les autres entretiens cités dans cet article, il a été retranscrit en annexe de ma thèse, La Justice dans la guerre d'Algérie, sous la dir. de J.-J. Becker, Université Paris X-Nanterre, 1999, vol. 3. Toutes les citations de P. Braun dans cet article sont tirées de cet entretien.

20. J. Vergès, entretien avec l'auteur.

21. M. Oussedik, entretien avec l'auteur. Toutes les citations de M. Oussedik dans cet article en sont tirées.

22. P. Kaldor, entretien avec l'auteur. Toutes les citations de P. Kaldor dans cet article en sont tirées.

23. P. Kaldor, entretien avec l'auteur. 
Dreyfus, Marcel Manville, Michel Bruguier, François Brault, Angeline Dominique ${ }^{24}$. C'est ainsi que Jacques Vergès, alors membre du PCF, partit plaider en Algérie pour la première fois en 1957. En désaccord avec les positions de son parti sur l'Algérie, il rompit ensuite avec lui et rejoignit le collectif de la Fédération de France du FLN au moment de sa création.

Les avocats métropolitains ne pouvaient pas assurer seuls la défense dans les procès tenus en Algérie : « Il fallait plaider tous azimuts : Alger, Blida, Constantine, Oran, etc. ", explique Pierre Braun. C'est pourquoi un collectif fonctionnait en Algérie, parallèlement au collectif métropolitain. Pierre Braun dit l'avoir créé non seulement pour " renforcer notre équipe " mais aussi pour des raisons politiques :

"On considère qu'il n'est pas normal, sur le plan politique comme sur le plan de l'organisation, que ce soient uniquement des avocats français qui défendent les militants victimes de la répression. Par conséquent, j’ai moimême constitué à Alger un collectif algérien avec une dizaine d'avocats comme Amar Bentoumi, devenu ministre de la Justice à l'indépendance, Guedj, Benmelha, Grange... [...] J'avais pris la précaution de faire en sorte que l'origine nationale de ces avocats soit à peu près équilibrée entre avocats musulmans et avocats d'origine européenne."

Il dément que ce collectif ait été un collectif communiste, " même s'il y a deux ou trois communistes parmi eux, sur onze ou douze ". Albert Smadja, du barreau d'Alger, en fit partie. Avant 1954, il avait été l'avocat du journal communiste Alger Républicain et avait défendu des militants communistes. Le fonctionnement du collectif d'Algérie s'apparentait à celui du collectif métropolitain : " On se répartissait les affaires et les inculpés. Par exemple, s'il y avait quinze inculpés dans une affaire, on prenait l'affaire à trois ", se souvient $\mathrm{M}^{\mathrm{e}}$ Smadja. Les demandes qui leur étaient adressées émanaient également de détenus qui les connaissaient de réputation : "Les gens venaient naturellement à nous car la prison est une caisse de résonance énorme. Il y a des jours, on recevait des dizaines et des dizaines de lettres ${ }^{25}$.

Préexistant au collectif de la Fédération de France du FLN, ces collectifs métropolitain et algérois se présentent comme des structures souples et extensibles, au fonctionnement pragmatique. Bien que liés au PCF en métropole et marqués par l'investissement des membres du PCA en Algérie, ces collectifs n'avaient pas une vocation politique prioritaire. Certes, l'intervention d'avocats engagés évitait aux militants de l'indépendance les déboires causés par l'hostilité de défenseurs émargeant aux barreaux locaux. "Certains se faisaient gloire de ne pas défendre des inculpés favorables à la cause de l'indépendance. D'autres faisaient des plaidoiries honteuses, c'est-à-dire qu'ils se défendaient d'être d'accord avec leurs clients ", raconte $\mathrm{M}^{\mathrm{e}} \mathrm{Smadja}$. En métropole, en outre, les avocats alimentaient le scandale de la répression : en janvier 1955, par exemple, Pierre Stibbe fournit à Claude Bourdet et à François Mauriac la matière de leurs articles parus dans France Observateur et L'Express, emblématiques de la précocité de la dénonciation de la torture ${ }^{26}$. Mais, sans nier la dimension politique de leur engagement, ceux qui témoignent a posteriori de

24. Liste des départs d'avocats conservée par P. Kaldor et communiquée lors de l'entretien précité.

25. A. Smadja, entretien avec l'auteur. Toutes les citations d'A. Smadja dans cet article en sont tirées.

26. C. Bourdet, «Votre Gestapo d'Algérie», France Observateur, 13 janvier 1955 ; F. Mauriac, «La question ", L'Express, 15 janvier 1955. 
ce passé insistent sur le fait que ces collectifs répondaient d'abord à une "nécessité pratique " : "L'objectif est de s'aider mutuellement et de s'organiser pour défendre nos clients ", précise Albert Smadja. L'ampleur de la répression judiciaire en Algérie l'explique. Elle explique aussi la difficulté à clore la liste des avocats métropolitains ayant plaidé en Algérie : à un noyau dur de fidèles très investis s'ajoutaient des défenseurs intervenant moins fréquemment et d'autres encore qui ne furent que ponctuellement sollicités, suivant les besoins.

Dans ce contexte, le collectif créé en 1958 par la Fédération de France du FLN innova radicalement. Selon Ali Haroun, ancien dirigeant de la Fédération de France, sa constitution résulta de désaccords irréductibles avec les avocats de la gauche française ${ }^{27}$. Le déclencheur en aurait été le refus de Pierre Stibbe, sollicité pour servir d'agent de liaison avec les cinq prestigieux prisonniers de la Santé, tous fondateurs du FLN : Ahmed Ben Bella, Hocine Aït Ahmed, Mohamed Khider, Mohamed Boudiaf et Rabah Bitat. Au-delà de ce cas précis, la Fédération de France concevait l'intervention des avocats comme une mobilisation au service de ses besoins. Elle reproduisait à l'égard des avocats métropolitains la logique d'exclusivité que le FLN avait généralement adoptée, refusant toute alliance avec d'autres organisations mobilisées contre la colonisation française en Algérie. De son point de vue, la lutte pour l'indépendance ne pouvait passer que par une intégration de militants en son sein. Mourad Oussedik raconte qu'il envisagea la participation d'avocats communistes au collectif nouvellement formé, mais qu'ils auraient dû s'y fondre individuellement. La création de ce collectif s'accompagna d'ailleurs d'une véritable lutte pour la prise en charge des détenus, appelés à déconstituer les autres avocats. "On a commencé à prendre les prisons, méthodiquement. On a pris d'abord tout Paris, puis on a débordé : Orléans, Reims, Amiens, Rouen... ", se souvient Mourad Oussedik. Pierre Braun confirme : "Les instructions du FLN pour nous déconstituer ont été suivies d'effets en France où toute la défense a été menée par Vergès et son groupe ». Aucune étude d'envergure n'existe sur la répression judiciaire en France métropolitaine mais il est évident que, l'activité nationaliste y étant elle-même de moindre ampleur, les tribunaux furent moins sollicités ${ }^{28}$. Les cours d'assises restèrent d'ailleurs compétentes jusqu'au 8 octobre 1958, date à laquelle les tribunaux militaires leur furent substitués, comme en Algérie.

La ligne de fracture séparant les avocats du collectif FLN des avocats " historiques " reproduit celle qui sépara les porteurs de valise des autres anticolonialistes français ${ }^{29}$. Il s'agissait de soutenir la lutte pour l'indépendance de l'Algérie en se mettant à disposition du FLN, dans un geste solidaire synonyme d'adhésion, au lieu d'agir dans un cadre autonome, ménageant une liberté d'action. Par rapport au RDA du début des années 1950, le FLN faisait franchir un degré supplémentaire à la collaboration entre une organisation victime d'une répression judiciaire et son

27. A. Haroun, La 7 wilaya, Paris, Seuil, 1986, p. 171.

28. Les études restent très partielles : A. Deperchin et A. Lecompte, "Les crimes commis par les Algériens en métropole devant la cour d'assises du Nord, 1954-1962 ", in La Justice en Algérie, Paris, La Documentation française, 2005, p. 257-270 ; C. LE GuEN, "La répression judiciaire : prétoires et prisons d'Ille-et-Vilaine ", in R. Branche et S. Thénault (dir.), La France en guerre. 1954-1962. Expériences métropolitaines de la guerre d'indépendance algérienne, Paris, Autrement, 2008, p. 340-355.

29. L'histoire de ce collectif est d'ailleurs retracée par H. Hamon et P. Rotman dans Les porteurs de valises. La résistance française à la guerre d'Algérie, Paris, A. Michel, 1979, $2^{\mathrm{e}}$ éd. augmentée, Seuil, 1982. 
collectif d'avocats. Du RDA au FLN, les relations entre mouvement nationaliste et défenseurs évoluaient de la concertation - avec risque de désaccord et de rupture - à l'établissement d'un lien organique. Sous la direction d'Aboubeker Belkaïd en 1960, le collectif de la Fédération de France fonctionna même « comme une véritable cellule du FLN $»^{30}$, relate Ali Haroun, chargé d'en suivre le fonctionnement et l'action au sommet. C'est dans un esprit de discipline révolutionnaire que le FLN réinvestit ce "mode d'organisation politico-professionnelle » ${ }^{31}$ qu'était le " collectif ", né dans les circonstances de la répression coloniale postérieure à 1945.

\section{Entre procès emblématiques et répression judiciaire de masse : quelles défenses?}

" Devant le tribunal correctionnel d'Alger, plusieurs prévenus, inculpés de délits mineurs (collecte de fonds pour le FLN, distribution ou colportage de tracts, etc.) ont déclaré ne pas reconnaître la compétence de la juridiction française ", relate $L e$ Monde dans son édition du 24 janvier 1957. L'article poursuit : "C'est la première fois que des prévenus ont une telle attitude mais il est à penser que de pareils moyens seront de nouveau employés ". Bien avant le collectif de la Fédération de France, qui en fit son credo, la négation de la compétence des juridictions françaises avait été préconisée par une directive du Comité de coordination et d'exécution (CCE), organe directeur du FLN à l'échelon central ${ }^{32}$. Il est cependant impossible d'évaluer l'emploi de cette argumentation devant les tribunaux, faute de pouvoir entrer dans leur enceinte a posteriori. Les livres et brochures publiés pour la défense de condamnés ne donnent accès qu'à quelques cas emblématiques ${ }^{33}$. À l'image de l'entrefilet du Monde précité, les comptes rendus de presse sont non seulement rares mais succincts. De leur côté, les avocats ne rédigent pas leurs plaidoiries.

En l'absence d'écrits, leurs souvenirs restent le meilleur guide pour tenter d'accéder aux salles d'audience. Interrogés une quarantaine d'années après, leur vocabulaire n'est pas celui de la "rupture " ou de la " connivence ", formalisées après la guerre d'indépendance algérienne. Ce qu'ils discutent, c'est la distinction schématique entre " plaider politique " et " plaider le dossier ». La première option - "plaider politique " - renvoie à toute stratégie consistant à user de l'arène judiciaire comme d'une arène médiatique et à transformer la défense d'accusés en celle d'une cause ; la seconde - "plaider le dossier " - désigne une défense consistant à argumenter en faveur des accusés sur le contenu du dossier et d'éventuels vices de procédure. Tous sont d'accord pour reconnaître que la différence n'était pas si marquée. Pierre Kaldor souligne que "Jacques Vergès et ses défenseurs étaient extrêmement habiles en matière de procédure, très fouilleurs, très attentifs aussi ", et Jacques Vergès reconnaît qu'il ne se privait pas de chercher les fautes de procédure afin d'enrayer le fonctionnement de la machine judiciaire.

Pierre Braun explique très simplement comment les deux types de plaidoiries pouvaient coexister : "Nous avons plaidé sur le plan politique mais également sur le plan des faits, dans la mesure où, quand nous avions la possibilité, sur la base

30. A. Haroun, La Te wilaya, op. cit., p. 177.

31. Pour reprendre l'expression de S. Elbaz dans « Les avocats métropolitains... ", art. cité.

32. J. Vergès y renvoie dans De la stratégie judiciaire, op. cit., p. 188-189.

33. Sur les affaires médiatisées : S. ThÉNAult, Une drôle de justice..., op. cit., p. 75-88 et p. 251-255. 
du dossier, de tirer d'affaire un certain nombre de gens, on ne s'en est pas privé ». $\mathrm{M}^{\mathrm{e}}$ Braun choisissait donc de plaider " politique » ou non suivant les circonstances : " Il est vrai que devant les tribunaux, nous avions des résultats qui étaient généralement assez médiocres, de telle sorte que, d'une fois sur l'autre, je changeais de tactique : quand j'avais plaidé politique, je me disais que la prochaine fois, je plaiderais sur les faits et, quand j'avais plaidé sur les faits, je me disais que la prochaine fois, je plaiderais politique puisque les inculpés sont des militants qui veulent qu'on exalte leurs idées et qu'ils y ont droit ".

Quant à Albert Smadja, il récuse l'idée d'une plaidoirie politique : "Tenir un discours politique ne servait à rien, les magistrats ne vous écoutaient pas ", et ce d'autant plus que "la multiplication des affaires provoque une dégradation de la défense politique, car on ne peut passer son temps à se répéter ». Il analyse le choix entre plaidoirie politique et plaidoirie sur le dossier de la même manière que Pierre Braun : "Si quelqu'un était évidemment coupable, on ne pouvait pas plaider le dossier, alors on plaidait politique. Mais si la culpabilité n'était pas évidente, on plaidait le dossier ». Pour lui, la répression judiciaire était en partie une parodie : "Tout cela n'avait aucune importance, sauf les condamnations à mort, car tout le monde savait que la guerre se terminerait et que la perpétuité ne le serait pas. L'objectif des avocats était donc surtout d'éviter les condamnations à mort ».

Précisément, c'est sur ce point que les divergences les plus graves s'expriment avec la stratégie que prônait Jacques Vergès. L'affaire Djamila Bouhired, une des poseuses de bombe de la bataille d'Alger, en est la parfaite illustration. Cette affaire fut la première plaidée par cet avocat dans le contexte algérien. Il était à cette date encore membre du PCF et partit à ce titre à Alger. Le procès fut émaillé d'incidents provoqués notamment par ses déclarations provocatrices : "Est-ce que sommes-nous ici dans un tribunal militaire ou à un meeting d'assassinat ? ", lança-t-il notamment ${ }^{34}$. Sommé de présenter des excuses au président du tribunal, Jacques Vergès refusa et reçut un avertissement. Interrompu par de multiples suspensions d'audience, le procès devint quasi-impossible et Jacques Vergès entreprit la campagne pour la grâce de Djamila Bouhired, condamnée à la peine capitale, en collaboration avec Georges Arnaud. Or, cette volonté de créer les incidents aurait eu pour contrepartie le sacrifice du sort de ses clients : "Ma stratégie de défense provoquait la condamnation à mort de beaucoup de mes clients mais, se défend-il, elle empêchait leur exécution en raison de la médiatisation de ces procès impossibles " ${ }^{35}$. Gisèle Halimi lui opposa une deuxième critique forte. Si cette stratégie avait l'avantage de capter l'attention médiatique, la succession d'incidents provoqués par les défenseurs contrariait le déroulement habituel des procès. Pour l'avocate, c'était se priver de l'occasion d'exploiter les audiences dans toutes leurs potentialités. Il fallait évidemment qu'elles aient lieu pour que les défenseurs usent du tribunal au service de leur cause, par l'audition des témoins, par la voix de leurs clients ainsi que par leurs plaidoiries. Il fallait qu'elles aient lieu pour que les accusés prennent corps dans toute leur humanité et sensibilisent l'opinion à leur cause. Gisèle Halimi abandonna ainsi la défense d'accusées au procès Jeanson, qui visait des porteurs de valise, traduits devant le tribunal militaire de Paris, en 1960. Elle regretta publiquement que les incidents

34. Voir les extraits des audiences reproduits dans G. Arnaud et J. Vergès, Pour Djamila Boubired, Paris, Éditions de Minuit, 1957, p. 48-49.

35. J. Vergès, entretien avec l'auteur. 
à répétition provoqués par ses confrères du collectif du FLN aient occupé les huit premiers jours d'audience ${ }^{36}$.

La médiatisation, enfin, imposait ses contraintes. Elle n'était possible qu'en métropole, où la presse se faisait l'écho de l'opposition à la guerre mobilisant des intellectuels et des militants de gauche. Elle ne pouvait concerner qu'un nombre limité de procès, dont les accusés présentaient un profil favorable. La lecture de la presse métropolitaine permet ainsi de repérer les filtres à l'œuvre entre la répression judiciaire de masse déployée en Algérie et la médiatisation des victimes. S’y prêtaient particulièrement bien les cas dans lesquels la culpabilité des accusés pouvaient être mise en doute, en particulier lorsqu'il s'agissait de condamnés à mort. En effet, pour l'opinion métropolitaine, sous le choc de la bataille d'Alger qui polarisa l'attention des médias en 1957, la guerre des Algériens, au quotidien, se présentait comme une suite d'attentats, d'assassinats ou d'embuscades militaires au cours desquelles des soldats français risquaient leur vie. L'engagement des militants de l'indépendance algérienne au service de leur cause et, qui plus est, leur choix de la lutte armée et du terrorisme, étaient des hypothèques à lever pour sensibiliser l'opinion à leur sort. Plaider l'innocence, c'était aussi rechercher la trace laissée par l'affaire Dreyfus dans la mémoire collective et toucher les intellectuels par l'évocation d'une tradition humaniste propre à la nation française. La torture occupait une place centrale dans cet argumentaire. La médiatisation, par conséquent, passait avant tout par une démonstration d'innocence des accusés, fondée sur la fragilisation des charges retenues contre eux, en raison des sévices qu'ils avaient subis. Dans une moindre mesure, elle passait aussi par une affirmation de la légitimité du nationalisme algérien, grâce à une analogie avec le nationalisme français, en particulier celui dont les résistants avaient fait preuve pendant la Seconde Guerre mondiale. Elle bénéficia, enfin, essentiellement à des femmes ou à des Européens.

Jacques Vergès explique ainsi que la médiatisation de Djamila Bouhired supposa de faire d'elle, " âme du terrorisme », le symbole des victimes. La jeune fille avait été blessée lors de son arrestation, puis torturée. Pour Jacques Vergès, la " jeune fille " qu'elle était pouvait " prêter son visage à la Révolution algérienne ". "C’est une chance pour elle. Elle peut être une locomotive pour cette Révolution ", insiste-t-il en tentant de reconstituer le processus de décision ayant abouti à la médiatisation de son $\operatorname{cas}^{37}$. Outre le nom de Djamila Bouhired résonnèrent en métropole ceux de Fernand Iveton, des époux Guerroudj, d'Abderrahmane Taleb ${ }^{38}$. Or, hormis celui d'Abderrahmane Taleb, ces quelques cas sont trompeurs : pendant la guerre d'indépendance, les condamnés à mort furent presque exclusivement des hommes, des Algériens dits «musulmans " selon le vocabulaire de l'époque, impliqués dans le terrorisme urbain ou dans les maquis. Seules six femmes furent condamnées à mort : Djohar Akrour, Baya Hocine, Djamila Bouazza, Djamila Bouhired, Jacqueline Guerroudj et Zahia Kherfallah ${ }^{39}$. Elles furent toutes graciées. Fernand Iveton, par ailleurs, resta le seul Européen condamné à mort qui fut exécuté. Outre le traitement différencié des accusés des deux sexes, traditionnel dans l’administration de la peine

36. H. Hamon et P. Rotman, Les porteurs de valise..., op. cit., p. 291.

37. J. Vergès, entretien avec l'auteur.

38. S. Thénault, Une drôle de justice..., op. cit., p. 75-88.

39. D’après D. Amrane, Les femmes algériennes dans la guerre, Paris, Plon, 1991, p. 159, note 2. 
capitale, la « coloration raciale ${ }^{40}$ des inégalités inhérentes à la situation coloniale se répercutait à la fois dans la répression judiciaire et dans sa médiatisation. Les affaires dont la connaissance franchit la Méditerranée n'offrent qu'une vision parcellaire et déformée de la répression judiciaire en terre algérienne.

Plus généralement, les procès cités en exemple de la stratégie de défense voulue par le FLN et incarnée a posteriori par Jacques Vergès, se sont pratiquement tous déroulés en métropole et ils sont peu nombreux. Sont mentionnés les procès des hommes impliqués dans la tentative d'ouverture en 1958 d'un " second front " en territoire métropolitain - procès de l'attaque de la Cartoucherie de Vincennes, de l'incendie de Mourepiane, de l'attentat contre Jacques Soustelle - ainsi que le procès du réseau Jeanson en $1960^{41}$. Joue d'évidence ici la vocation métropolitaine du collectif de la Fédération de France du FLN. Par ailleurs, le nombre de procès cités reste limité car la "défense politique " confinant à la " rupture " ne se conçoit pas, par nature, dans la répétition. C'est une défense qui suppose un investissement sur des affaires susceptibles d'être montées en épingle et de franchir les filtres de la médiatisation. Elle offre ainsi une réponse à une répression judiciaire de masse, dans le sens où elle permet d'attirer l'attention sur cette répression à travers quelques cas emblématiques. Mais elle ne constitue pas une stratégie à développer systématiquement dans tous les procès. Elle n'épuise donc pas la connaissance de la défense des nationalistes algériens. Au barreau lyonnais, ainsi, la défense de rupture était loin d'être systématiquement pratiquée par les avocats prenant en charge ces affaires ${ }^{42}$.

C'était évidemment aussi - et surtout - le cas dans la colonie elle-même. Sur place, les comptes rendus d'audience du tribunal militaire de Tiaret, en 1960-1961, témoignent d'un fonctionnement routinisé, sans éclat. En deux ans, le policier des Renseignements généraux chargé de suivre les audiences ne remarqua qu'une légère évolution : "L'élément féminin vient de plus en plus assister aux séances de ce tribunal, ainsi qu'un nombre croissant de jeunes " ${ }^{43}$. Trois audiences seulement eurent quelque retentissement. La première vit se succéder dans le box cinq membres de l'organisation politico-administrative (OPA) du FLN puis trois déserteurs, dont deux harkis. La deuxième fut en grande partie occupée par le procès du chef de l'Armée de libération nationale (ALN) locale, Hamdani Adda ${ }^{44}$. Ces deux audiences ne se distinguent toutefois des autres que par l'affluence du public. La troisième qui retint l'attention du policier concernait le frère d'un adjoint au maire d'une commune de la région. Lui aussi condamné à mort, il fut l'auteur du seul "incident » rapporté :

40. Selon l'expression de G. Batandier dans « La situation coloniale : approche théorique », Cahiers internationaux de sociologie, vol. 11, 1951, p. 73.

41. Ces procès sont mentionnés par A. Haroun dans La 7 e wilaya, op. cit., p. 169-183, et par J. Vergès dans De la stratégie judiciaire, op. cit., p. 192-194 ainsi que dans L'anticolonialiste, entretiens avec Philippe Kraim Felissi, Paris, Le Félin, 2005, p. 57 sq.

42. A. Grosjean, "Les avocats lyonnais et la défense des indépendantistes ", in B. Dubell, A. Grosjean et M. Thivend (dir.), Récits d'engagements : des Lyonnais auprès des Algériens en guerre, 1954-1962, Paris, Bouchène, 2012, p. 79-96.

43. Compte rendu de l'audience du 25 octobre 1960, Service historique de la Défense (SHD), $1 \mathrm{H} \mathrm{4026/1} \mathrm{(consultation} \mathrm{sous} \mathrm{dérogation).} \mathrm{L'ensemble} \mathrm{des} \mathrm{comptes} \mathrm{rendus} \mathrm{relatifs} \mathrm{aux} \mathrm{audiences} \mathrm{du}$ 23 janvier 1960 au 26 janvier 1962 est conservé sous cette cote.

44. Condamné à mort, il connut une fin tragique : alors que les exécutions avaient cessé, il fut enlevé de la prison par un commando de l'OAS qui le brûla vif, dans une logique de substitution aux pouvoirs publics accusés de laxisme. Voir A. BeLkHodja, L'affaire Hamdani Adda, brûlé vif par l'OAS, Tiaret, Éd. Mekkloufi, s. d., rééd. Alger, Éd. Enag, 2009. 
"Après que sa sentence eut été prononcée, l'inculpé s'est écrié à plusieurs reprises "Yahia el Djazair !" ${ }^{5}$ et les membres de sa famille lui ont répondu : "courage" " 46. Les défenseurs, quant à eux, ne suscitèrent aucun commentaire. Il s'agissait invariablement d'un avocat et de deux avoués locaux, ainsi que d'officiers défenseurs attachés au tribunal. L'organisation de la défense semble en outre avoir été rationalisée. Alors qu'au début de l'année 1960, plusieurs défenseurs intervenaient au cours d'une journée, il ne figure plus qu'un seul nom dans chaque compte rendu après février 1961, ce qui semble signifier qu'à partir de cette date, un seul défenseur était commis d'office pour toutes les affaires inscrites à l'audience du jour. Albert Smadja se souvient des officiers défenseurs : "On avait fait appel à des volontaires, en général des avocats, qui acceptaient d'être mobilisés avec, habituellement, le grade de capitaine. Ils étaient chargés d'assurer la défense de ceux qu'on leur présentait ». Depuis l'avènement de la $V^{e}$ République, les réformes gaullistes avaient approfondi la militarisation du fonctionnement de la justice en Algérie. L'instruction avait été supprimée, au profit d'une enquête rapidement menée par un magistrat placé sous les drapeaux. Aucun avocat n'intervenait dans cette phase antérieure au renvoi devant le tribunal. Les tribunaux correctionnels avaient en outre été totalement dessaisis. Accusés de crimes ou de délits, les nationalistes algériens étaient tous passibles de la juridiction militaire. Dans ce contexte, les tribunaux militaires avaient été multipliés, passant de trois à quinze - l'existence d'un tribunal à Tiaret, à deux cents kilomètres au sud d'Oran, résultait de cette décentralisation. La possibilité de faire appel à des officiers défenseurs avait été ouverte à ce moment-là. Près de seize mille personnes furent jugées dans ces conditions ${ }^{47}$. La défense n'était plus que de pure forme.

Le rôle des avocats des barreaux locaux, français comme algériens - et la façon dont ils défendirent les nationalistes - reste le point aveugle de cette histoire. Dans la mémoire de Pierre Kaldor, certains, des " avocats de famille », en particulier, "prononçaient des plaidoiries tout à fait inadéquates du type : "M. le juge, c'est une femme, elle a été entraînée, etc.” Je vous donne cet exemple-là parce qu'une fois, une femme révoltée m’a demandé en pleine audience de suppléer son avocat ". Cette femme était une responsable du FLN et Pierre Kaldor était venu assister d'autres accusés. Le rôle de tels avocats, pourtant, est d'autant plus important que les autorités multiplièrent les entraves à l'exercice de la profession par leurs confrères engagés.

\section{Les droits du défenseur : un enjeu}

La distinction entre métropole et Algérie doit être d'autant plus rigoureuse que la circulation entre les deux territoires n'était pas libre. Les avocats des barreaux métropolitains devaient demander l'autorisation de se rendre en Algérie et les autorités ne se privèrent pas de la leur refuser. Marcel Manville, par exemple, batailla jusqu'au Conseil d'État contre un refus qui lui interdit d'assister deux de ses clients condamnés à mort, au moment de leur exécution ${ }^{48}$. Des interdictions de séjour furent aussi

45. "Vive l'Algérie!"

46. Compte rendu de l'audience extraordinaire du 8 décembre 1961, SHD, 1H 4026/1 (sous dérogation).

47. S. Thénault, Une drôle de justice..., op. cit., p. 220.

48. S. ThÉnault, "La guerre d'Algérie au Conseil d'État ", in J. Massot (dir.), Le Conseil d'État et l'évolution de l'outre-mer français du XVIII' siècle à 1962, Paris, Dalloz, 2007, p. 199-220. 
prononcées. L'ampleur de ces mesures individuelles est impossible à évaluer mais les défenseurs métropolitains engagés en font état à leur encontre. "Après 1958, j’ai été complètement interdit de séjour ", rapporte Pierre Braun, tandis que Pierre Kaldor se dit « interdit d'Algérie après 1960 ». Tous deux précisent néanmoins que des confrères continuèrent à aller plaider en Algérie. Il importerait de vérifier dans quelle mesure ces refus et interdictions ont globalement contrarié l'accès des avocats de métropole au territoire algérien. Les restrictions s'appesantirent-elles avec le temps ? Fluctuèrent-elles au gré de l'intensité de l'affrontement entre les Français et leurs adversaires ? Elles accrurent en tout cas l'importance des avocats inscrits aux barreaux locaux dans la défense des militants de l'indépendance sur place.

Or ces avocats furent aussi pris pour cible. En février 1957, onze avocats du barreau d'Alger, membres du collectif local, dont Albert Smadja faisait partie, furent arrêtés et internés, à la demande du commandement du général Massu. Ces avocats passèrent de plusieurs mois à plus d'un an dans les deux camps où ils avaient été conduits : celui de Lodi, réservé aux "Européens ", et celui de Berrouaghia destiné aux «musulmans " ${ }^{49}$. Leurs arrestations et détentions mobilisèrent les plus éminents représentants de la justice et du barreau en Algérie. Le procureur général d'Alger, Jean Reliquet, réaffirmait la légitimité des prérogatives judiciaires bafouées par une décision reposant sur des renseignements de source militaire. Sans motivation précise et en dehors de toute procédure contradictoire, les arrêtés d'internement étaient signés par les autorités préfectorales. Le procureur général ouvrit alors une information judiciaire afin d'obtenir communication des pièces versées au dossier. En juriste rigoureux qui se concevait comme un gardien des libertés publiques, il contesta la valeur probante des documents fournis par les militaires, faute d'en obtenir l'original ou les procès-verbaux authentifiant les circonstances de leur découverte : il s'agissait de reproductions photographiques de trois documents du FLN. Le bâtonnier Perrin, du barreau d'Alger, se porta quant à lui garant de l'intégrité professionnelle et personnelle des internés. À Paris, par ailleurs, le bâtonnier Thorp protesta auprès du président de la République. Cette mobilisation au nom du respect des libertés publiques et de la déontologie jouait sur une argumentation paradoxale. « Le bâtonnier Perrin, bâtonnier d'Alger, est venu me voir au commissariat et m'a annoncé qu'il avait fait un communiqué de presse en affirmant que mon arrestation n'avait rien à voir avec mes activités professionnelles, relate Albert Smadja. Il pensait m’avoir rendu service alors qu'en réalité, nous avions été arrêtés exclusivement en raison de nos activités professionnelles. »

Dans le contexte d'une guerre qui put être comparée à une gigantesque affaire Dreyfus, les appels au respect des principes généraux du droit, du fonctionnement de la justice et des droits de la défense ne manquèrent pas et ils furent précoces. Dès janvier 1956, des avocats du barreau de Constantine avaient dénoncé les conditions de la répression en Algérie, réduisant le "rôle de l'avocat " à une " assistance tardive et illusoire " ${ }^{50}$. Évidemment conscientes de la ressource argumentaire et concrète que constituaient les droits des défenseurs, les autorités françaises les restreignirent progressivement - l'état d'urgence limitait déjà les recours possibles contre les décisions d'instruction. In fine, l'ordonnance du 6 octobre 1960 contre-attaqua en visant précisément la " défense politique " et la stratégie incarnée par Jacques

49. S. ThÉnault, Une drôle de justice..., op. cit., p. 115-118.

50. Déclaration reproduite dans Le Monde et L'Humanité du 13 janvier 1956. 
Vergès. Quelques jours après la fin du procès Jeanson, cette ordonnance concernait uniquement les procès tenus devant les tribunaux militaires. Elle limitait les auditions de témoins aux faits ou à la personnalité de l'accusé et interdisait toute notification de nouveaux témoins en cours de débats. Elle épargnait aux tribunaux militaires l'examen des conclusions déposées par les avocats avant d'ouvrir le procès lui-même - car le dépôt de conclusions à répétition, soulevant des questions de procédure, était l'un des moyens utilisés par les avocats du collectif du FLN pour contrarier le déroulement des audiences. L'ordonnance prévoyait enfin qu'en cas de " manquement aux obligations " de leur serment, les avocats pourraient être sanctionnés immédiatement par le tribunal militaire. Ces sanctions prirent la forme d'une interdiction d'exercer - Jacques Vergès la subit ainsi pendant trois mois par décision du tribunal militaire de Paris, le 11 octobre $1960^{51}$. Entre autres sanctions, il fut aussi suspendu pendant un an par le conseil de l'ordre de la cour d'appel de Paris, à compter du 8 janvier 1961. Ses confrères du collectif FLN subirent des sanctions similaires. Ils furent aussi poursuivis en justice pour atteinte à la sûreté de l'État et deux d'entre eux, $\mathrm{M}^{\text {es }}$ Oussedik et Benabadallah, furent internés ${ }^{52}$.

Dans ce contexte, quel rôle jouèrent les conseils de l'ordre ? Ce rôle oscille potentiellement entre deux pôles : un rôle de vigie au service de la préservation des droits des défenseurs, que personnalisent, par exemple, les bâtonniers Perrin et Thorp s'élevant contre l'internement des avocats algérois en 1957 ; un rôle disciplinaire contre tout usage de ces droits perçus comme abusifs, tel le conseil de l'ordre parisien sanctionnant les avocats du collectif du FLN. Concrètement, leur appui fut-il une ressource ou leur rigueur constitua-t-elle un frein dans la défense des militants de l'indépendance ? Les barreaux d'Algérie seraient particulièrement à interroger. Au-delà de leurs minorités engagées dans la défense des nationalistes, repérables dès les procès de l'OS avant-guerre et pendant la guerre elle-même, leurs membres se firent connaître par leur parti pris colonial. Après la semaine des Barricades, en janvier 1960, au cours de laquelle les partisans de l'Algérie française mirent en état d'insurrection le centre d'Alger, deux avocats algérois, dont le propre fils du bâtonnier, furent poursuivis pour leur activisme. Or le conseil de l'ordre protesta. Il appela au boycott de la cérémonie d'installation du nouveau procureur général d'Alger, choisi pour son loyalisme à l'égard du général de Gaulle et d'une politique prenant le chemin de la négociation avec les Algériens. L'appel du conseil de l'ordre rencontra un vif succès : seule une vingtaine d'avocats, sur un total de 280, assistèrent à cette cérémonie. Par la suite, le fils du bâtonnier, Jacques Laquière, rejoignit le général Salan à Madrid, où l'Organisation de l'armée secrète (OAS) fut créée au début de l'année 1961 dans le milieu des activistes français ayant trouvé refuge en terre franquiste. Le conseil de l'ordre des avocats d'Alger, enfin, fut dissous après la tentative de putsch d'avril 1961. Assistant à l'arrestation du procureur général d'Alger par des officiers insurgés, plusieurs d'entre eux n'auraient pas réagi. Le conseil de l'ordre ne fut rétabli que six mois plus $\operatorname{tard}^{53}$.

51. Sur ces sanctions, voir S. ThÉNAult, Une drôle de justice..., op. cit., p. 227. Les sanctions contre J. Vergès furent récapitulées par le ministre des Armées, Pierre Messmer, dans une lettre adressée au commandant en chef des forces françaises en Algérie, le 30 janvier 1961. SHD, 1H 1097/4 (sous dérogation).

52. Sur l'internement de ces avocats, voir A. Grosjean, " $\mathrm{M}^{\mathrm{e}}$ Abdessamad Benabdallah à Thol ", Matériaux pour l'histoire de notre temps, ${ }^{\circ} 92$, octobre-décembre 2008, p. 48.

53. S. Thénault, Une drôle de justice..., op. cit., p. 209 et p. 285. 
Ainsi abordée à travers une focalisation sur le politique et sur la période finale de l'indépendance, moment de radicalisation, l'histoire des avocats d'Algérie dessine un mouvement inverse à celui de l'historiographie métropolitaine. En métropole, les avocats ont été décrits dans leur rôle social avant de l'être dans leurs engagements minoritaires ${ }^{54}$. Cet article ne peut se terminer que sur un appel à l'histoire des barreaux d'Algérie, appréhendés dans leur composition sociale et dans leur ancrage au sein de la société coloniale.

L'image dominante d'une défense assurée par un collectif d'avocats plaidant à la demande du FLN, en "rupture » avec les normes du fonctionnement de la justice, doit ainsi être triplement relativisée. Le collectif de la Fédération de France du FLN ne fut évidemment pas le seul et il ne fut pas créé dès le début de la guerre. La "défense de rupture " ne fut conceptualisée qu'a posteriori et elle reste impuissante à décrire concrètement la façon dont les avocats des militants de l'indépendance assurèrent leur défense. L'ampleur de la répression judiciaire en territoire algérien, enfin, impliqua l'intervention non seulement d'avocats locaux dénués de préoccupations politiques mais aussi d'officiers défenseurs, commis d'office, assurant à leurs clients une défense de pure forme. Cette mise au point serait cependant vaine si elle n'ouvrait pas des pistes pour l'avenir. C'est d'abord un déplacement du questionnement qui a été proposé : il s'agit moins de savoir ce que représente la séquence de la guerre d'indépendance dans l'histoire de l'engagement des avocats que de se demander comment, concrètement, les nationalistes algériens furent défendus. Ce sont ensuite les barreaux locaux, en Algérie, qui sont apparus comme le lieu privilégié de l'observation et de l'analyse de cette défense. Leur histoire serait néanmoins à réinscrire dans la longue durée, dans le temps ordinaire de la colonisation française en Algérie.

54. À travers la problématique du cause lawerying, appliquée au terrain français, voir B. GAÏTI et L. IsraëL, "Sur l'engagement du droit dans la construction des causes ", Politix, vol. 16, n62, 2003, p. 17-30. 